\title{
Comparison of Young and Adult Rat Models of Chemotherapy- Induced Alopecia
}

\begin{abstract}
Keywords: Anagen; Rat model; Heat shock; Chemotherapy induced alopecia

Abstract

Background: Chemotherapy-induced alopecia (CIA) is a common side effect of cancer treatment with profound psychosocia repercussions. Several young (before day 14 ) and a dult (after 3 weeks) murine models for CIA have been developed, yet no study thusfar has compared these models. Young models a re naturally synchronized in anagen while adult models require synchronization into a na gen. Here, we compared the gross and histological features of the young versus adult models of CIA in pigmented rats. Additionally, we determined the effectiveness of heat shock treatment to prevent CIA; heat shock has not been tested before in pigmented young or adult rats.
\end{abstract}

Methods: To induce synchronized anagen, adult rats were clipped during early telogen. To induce a lopecia, young and a dultratsreceived intraperitoneal injections of etoposide or cyclophosphamide. Rats from each model were randomized to receive heat shock preceding chemotherapy. Alopecia was observed 10 days post-chemotherapy, and dorsal skin biopsies were analyzed for histology.

Results: Upon chemotherapy, young rats developed tota alopecia, whereas adult rats developed alopecia restricted to the clipped area. Histologically, equivalent hair follicle dystrophy was observed in each model 10 days post-chemotherapy. Moreover, both the young and adult rats demonstrated areas of protection from CIA corresponding to localized heat shock pretreatment.

Conclusions: Both the young and adult rat models mimic clinica and microscopic findings of $\mathrm{ClA}$. However, the young rat model, with naturally synchronized anagen, develops total alopecia upon chemotherapy, and may be a more suitable model to screen protective compounds.

\section{Abbreviations}

CIA: Chemotherapy induced alopecia; HF: Hair follicle; heat shock protein 70i (HSP70i, HSPA1A)

\section{Introduction}

Alopecia is one of the most distressing and frequent side effects of many antineoplastic regimens. It is estimated that approximately $65 \%$ of patients treated with chemotherapy experience some degree of hair loss $[1,2]$. Chemotherapy induced alopecia (CIA) is often reversible; however, cases of permanent alopecia have been reported [3]. In more than $60 \%$ of patients, initial regrown hair is of a different texture or color [3]. The psychological impact of CIA can be devastating, as it negatively affects a patient's self-esteem and removes one's health related confidentiality [4-8]. To date, no treatment has been found to reliably and completely prevent CIA in humans.

Most of what is known about the pathophysiology of CIA has been gained through experimentation with either young (corresponding to the first postnatal anagen) or adult (after the first postnatal anagen)

\section{Clinical \& Investigative Dermatology}

\author{
Villasante $\mathrm{AC}^{1}$, Wikramanayake $\mathrm{TC}^{1}$, Mauro $\mathrm{LM}^{1,2}$, \\ Yin NC ${ }^{1}$, Elgart GW $^{1}$, Schachner LA ${ }^{1}$ and Jimenez \\ $\mathbf{J J}^{1,2 *}$ \\ ${ }^{1}$ Department of Dermatology and Cutaneous Surgery, University of \\ Miami Miller School of Medicine, USA \\ ${ }^{2}$ Department of Biochemistry and Molecular Biology, University of \\ Miami Miller School of Medicine, USA \\ *Address for Correspondence \\ Joaquin J Jimenez, Department of Dermatology and Cutaneous Surgery, \\ University of Miami Miller School of Medicine, 1600 NW 10th Avenue, \\ RMSB, Room 2063A, Miami, FL 33136 USA, Tel: 305-243-6586; Fax: 305- \\ 243-6191; E-mail: j.jimenez@med.miami.edu \\ Submission: 11 March 2014 \\ Accepted: 22 May 2014 \\ Published: 26 May 2014 \\ Reviewed \& Approved by: Dr. Nihal Ahmad, Professor in \\ Department of Dermatology at the University of Wisconsin, USA
}

murine models using alopecic chemotherapies. The young Sprague Dawley albino rat model of CIA was the first murine model to mimic the alopecic effect of chemotherapy [9]. Other models such as the adult depilated C57BL/6 mouse model [10], the young C57BL/6 mouse model [11], and the adult Long Evans pigmented rat model [12] were subsequently developed. Importantly, murine models also provide a means of testing prospective protective agents against CIA.

The hair follicle (HF) undergoes cycles of anagen, catagen, and telogen $[1,13]$. Late anagen is further subdivided into six stages, the last of which is the time of most rapid growth, and consequently most susceptible to chemotherapy. In the human scalp, at any given time, about $90 \%$ of HFs are in anagen, and each follicle cycles autonomously $[14,15]$. In murine models, as extrapolated from studies in C57BL/6 mice, all dorsal and ventral HFs on the trunk undergo synchronized growth until postnatal day 14 (P14) $[16,17]$. After a short catagen, by P19-27, most pelage HFs are in telogen; only $10 \%$ are in anagen [10]. In subsequent hair cycles, HFs are no longer synchronized. Thus, to mimic the human scalp, chemotherapy is usually administered between P11 and P13 in the young murine models, and clipping or depilation is required in adult murine models for anagen synchronization prior to chemotherapy. The adult rat model is clipped during early telogen on P21 and chemotherapy is administered between P36 and 38 [12].

In the rapidly expanding field of CIA, it is imperative to determine which models are most useful to readily screen multiple potential treatment modalities. A proper model is one that mimics human HFs reliably. In this study, we directly compared young and adult pigmented rat models of CIA. Additionally, we applied heat shock treatment in the pigmented young and adult rat models to compare the response.

\section{Materials and Methods}

Animals and induction of chemotherapy-induced alopecia

All animal care and use procedures were approved by the University 
Citation: Villasante AC, Wikramanayake TC, Mauro LM, Yin NC, Elgart GW, et al. Comparison of Young and Adult Rat Models of ChemotherapyInduced Alopecia. J Clin Investigat Dermatol. 2014;2(2): 7.

of Miami Institutional Animal Care and Use Committee (IACUC). Nursing Long-Evans rat neonates (Charles River Laboratories, Wilmington, MA, USA) were maintained under standard conditions. To induce CIA in the young rats, rats were injected intraperitoneally (i.p.) with chemotherapeutic agents (Sigma-Aldrich, St. Louis, MO, USA): etoposide $(1.5 \mathrm{mg} / \mathrm{kg})$ once daily on postpartum day 11 (P11), 12, and 13; or cyclophosphamide $(37.5 \mathrm{mg} / \mathrm{kg})$ once on P13. Normal saline was injected on P11, 12, 13 in control rats. To induce CIA in the adult rats, first dorsal hair was clipped on P21 [12], after rats were anesthetized with ketamine/xylazine (50 mg/kg and $5 \mathrm{mg} / \mathrm{kg}$, SigmaAldrich, St. Louis, MO, USA). Caution was taken not to abrade the skin with the electric clipper. Rats were subsequently i.p. injected to induce alopecia: etoposide $(25 \mathrm{mg} / \mathrm{kg}$ ) once daily on P36, 37, 38; or cyclophosphamide $(75 \mathrm{mg} / \mathrm{kg})$ on P38. Normal saline was injected on P36, 37, 38 in control rats (vehicle). Animals were euthanized according to IACUC procedures.

\section{Prevention of chemotherapy-induced alopecia in the young rat model}

On P9, 80 rats were randomized into eight groups of 10 . The first four groups, a d, received heat shock treatment prior to chemotherapy, as described [11] with modifications. Briefly, heat was applied to the dorsal skin using a copper cylinder, which circulated heated water at $48^{\circ} \mathrm{C}$ for 20 minutes [11]. Subsequently, Groups a d received chemotherapy as follows: (a) etoposide; (b) cyclophosphamide; (c) normal saline; and (d) not injected (reference for weight and histopathology, analyzed on P11). The last four groups of rats, Groups 1 4, were not treated with heat shock but were similarly injected (Table 1). Group 4 was analyzed on P13.

\section{Prevention of chemotherapy-induced alopecia in the adult rat model}

One-hundred and twenty weaning rats were randomized into twelve groups of 10 on P21. Four of these groups (A D) were clipped on the dorsum, and on P34 they received heat shock treatment as described above for young rats. Subsequently, they received chemotherapy as follows: (A) etoposide; (B) cyclophosphamide; (C) normal saline; (D) no injection (used as reference for weight and histology, analyzed on P36).

The last eight groups (I VIII) did not receive heat shock treatment. Some groups were clipped on P21 and others went unclipped; and were subsequently injected with chemotherapy (Table 2) as follows: (I) clipped + etoposide; (II) not clipped + etoposide; (III) clipped + cyclophosphamide; (IV) not clipped + cyclophosphamide; (V) clipped + normal saline; (VI) not clipped + normal saline; (VII) clipped, no injection; and (VIII) not clipped, no injection. Groups VII and VIII were analyzed on P38 for histology.

\section{Sample collection, histological analysis, and immunohistochemistry}

Rats were monitored daily for signs of hair loss. Alopecia was scored 10 days post-chemotherapy by two independent observers

Table 1: Induction and prevention of alopecia development in young rats.

\begin{tabular}{|c|c|c|c|c|c|}
\hline \multirow{2}{*}{ Group } & \multirow{2}{*}{$\begin{array}{l}\text { Heat } \\
\text { (P9) }\end{array}$} & \multirow{2}{*}{$\begin{array}{l}\text { Injection } \\
\text { (P11 13) }\end{array}$} & \multicolumn{2}{|c|}{ Alopecia (on P21) } & \\
\hline & & & All hair preserved & Total alopecia & \\
\hline $\mathrm{a}$ & Heat & Etoposide & 10 & 0 & \multirow{2}{*}{\}$p<<0.01$} \\
\hline 1 & No heat & Etoposide & 0 & $10^{*}$ & \\
\hline $\mathrm{b}$ & Heat & Cyclophosphamide & 10 & 0 & \multirow{2}{*}{\}$p<<0.01$} \\
\hline 2 & No heat & Cyclophosphamide & 0 & $10^{*}$ & \\
\hline c & Heat & Normal Saline & 10 & 0 & \multirow{2}{*}{\}$p=1.00$} \\
\hline 3 & No heat & Normal Saline & 10 & 0 & \\
\hline$d$ & Heat & \multicolumn{3}{|c|}{ No injection, analyzed on P11 } & \\
\hline 4 & No heat & \multicolumn{3}{|c|}{ No injection, analyzed on P13 } & \\
\hline
\end{tabular}

${ }^{*} \mathrm{p}<<0.01$ compared with saline-injected young rats (Group 3)

Table 2: Induction and prevention of alopecia development in adult rats.

\begin{tabular}{|c|c|c|c|c|c|c|c|}
\hline \multirow{2}{*}{ Group } & \multirow{2}{*}{ Clipped (P21) } & \multirow{2}{*}{ Heat (P34) } & \multirow{2}{*}{$\begin{array}{l}\text { Injection } \\
\text { (P36 38) }\end{array}$} & \multicolumn{2}{|c|}{ Alopecia (on P47) } & & \\
\hline & & & & All hair preserved & Total alopecia & & \\
\hline A & Clipped & Heat & Etoposide & 10 & 0 & & \multirow{2}{*}{\}$p<<0.01$} \\
\hline 1 & Clipped & No heat & Etoposide & 0 & $10^{*}$ & \multirow{2}{*}{\}$p<<0.01$} & \\
\hline II & Not clipped & No heat & Etoposide & 10 & 0 & & \\
\hline B & Clipped & Heat & Cyclophosphamide & 10 & 0 & & \multirow{2}{*}{\}$p<<0.01$} \\
\hline III & Clipped & No heat & Cyclophosphamide & 0 & $10^{*}$ & \}$p<<0.01$ & \\
\hline C & Clipped & Heat & Normal Saline & 10 & 0 & & \\
\hline V & Clipped & No heat & Normal Saline & 10 & 0 & & \\
\hline VI & Not clipped & No heat & Normal Saline & 10 & 0 & & \\
\hline $\mathrm{D}$ & Clipped & Heat & \multicolumn{3}{|c|}{ No injection, analyzed on P36 } & & \\
\hline VII & Clipped & No heat & \multirow{2}{*}{\multicolumn{3}{|c|}{ No injection, analyzed on P38 }} & & \\
\hline VIII & Not clipped & No heat & & & & & \\
\hline
\end{tabular}

${ }^{*} \mathrm{p}<<0.01$ compared to saline-injected clipped adult rats (Group V) 
Citation: Villasante AC, Wikramanayake TC, Mauro LM, Yin NC, Elgart GW, et al. Comparison of Young and Adult Rat Models of ChemotherapyInduced Alopecia. J Clin Investigat Dermatol. 2014;2(2): 7.

and analyzed using Fisher's exact test; loss of $>90 \%$ pelage hair was considered "total alopecia." Dorsal skin biopsies were collected for histological analysis. Rats that did not receive chemotherapy or saline injections served as negative controls to their counterparts receiving chemotherapy to verify hair cycle stage.

Dorsal skin biopsies were collected and fixed in $10 \%$ formalin, dehydrated and embedded in paraffin wax, and sectioned at $5 \mu \mathrm{m}$. Hematoxylin and eosin staining was performed.

To analyze HSP1A1 (HSP70i) expression by immunohistochemistry, sections were incubated with mouse monoclonal anti-HSP70 antibody SPA-810 (Assay Designs, Ann Arbor, MI, USA), horse anti-mouse IgG biotinylated antibody (Vector Laboratories), and horseradish peroxidase avidin complex (Vector Laboratories), after heat-induced epitope retrieval. Signal was detected via incubation with $\mathrm{H}_{2} \mathrm{O}_{2}$ and 3,3'-diaminobenzidine. Counterstaining was performed with Mayer's hematoxylin. Images were captured using an Observer D1 microscope using 4x and 10x objectives (Carl Zeiss Microimaging Inc., Thornwood, NY, USA). Relative HSP level was measured using Western densitometry as previously described [18].

\section{Results}

Effects of etoposide and cyclophosphamide on hair follicles in the young rat vs. adult rat

Alopecia was observed in both the young rats and the clipped adult rats that were treated with chemotherapy. All young rats injected with either etoposide (a topoisomerase inhibitor) (Group
1)(Figure 1a) or cyclophosphamide (an alkylating agent)(Group 2) developed total body alopecia (except for vibrissae, some facial hair, and hair on the front paws) by 10 days post-chemotherapy (Table 1). All clipped adult injected with either etoposide (Group I)(Figure 1c) or cyclophosphamide (Group III) developed alopecia by 10 days after chemotherapy, localized to the clipped areas (Table 2). In unclipped adult rats, no hair loss was observed after injection with etoposide (Group II) or cyclophosphamide (Group IV) $(\mathrm{p}<<0.01)$ (Table 2). In both the young and adult models, no obvious hair loss was observed in the saline-injected (Groups 3, V)(Figure 1b, Figure 1d) and noninjected groups (Groups 4, VII) $(\mathrm{p}<<0.01)$ (Tables 1 and 2). These results are consistent with our previous studies [12].

HFs from non-injected young (Group 4) and adult (Groups VII and VIII) rats were assessed histologically to determine the hair cycle stage at the time of chemotherapy administration. In both young and clipped adult rats, HFs were found to be in anagen VI (Figure $2 \mathrm{a}$, Figure $2 \mathrm{~b}$ ). In contrast, HFs from unclipped rats on P38 were predominantly in anagen $\mathrm{V}$ (data not shown)[15].

Histological analysis was performed 10 days after chemotherapy to compare the HF response of young versus adult models of CIA. Skin obtained from rats from both models showed similar shortening of HFs and ectopic melanin granule accumulation (Figure 2c, Figure 2d), consistent with dystrophic catagen [15]. Additionally, only fragments of the hair shafts remain in the aberrantly widened hair canals. Extensive follicular deformation was noted. Furthermore, in both models, evident epidermal hyperplasia was observed in response to chemotherapy.

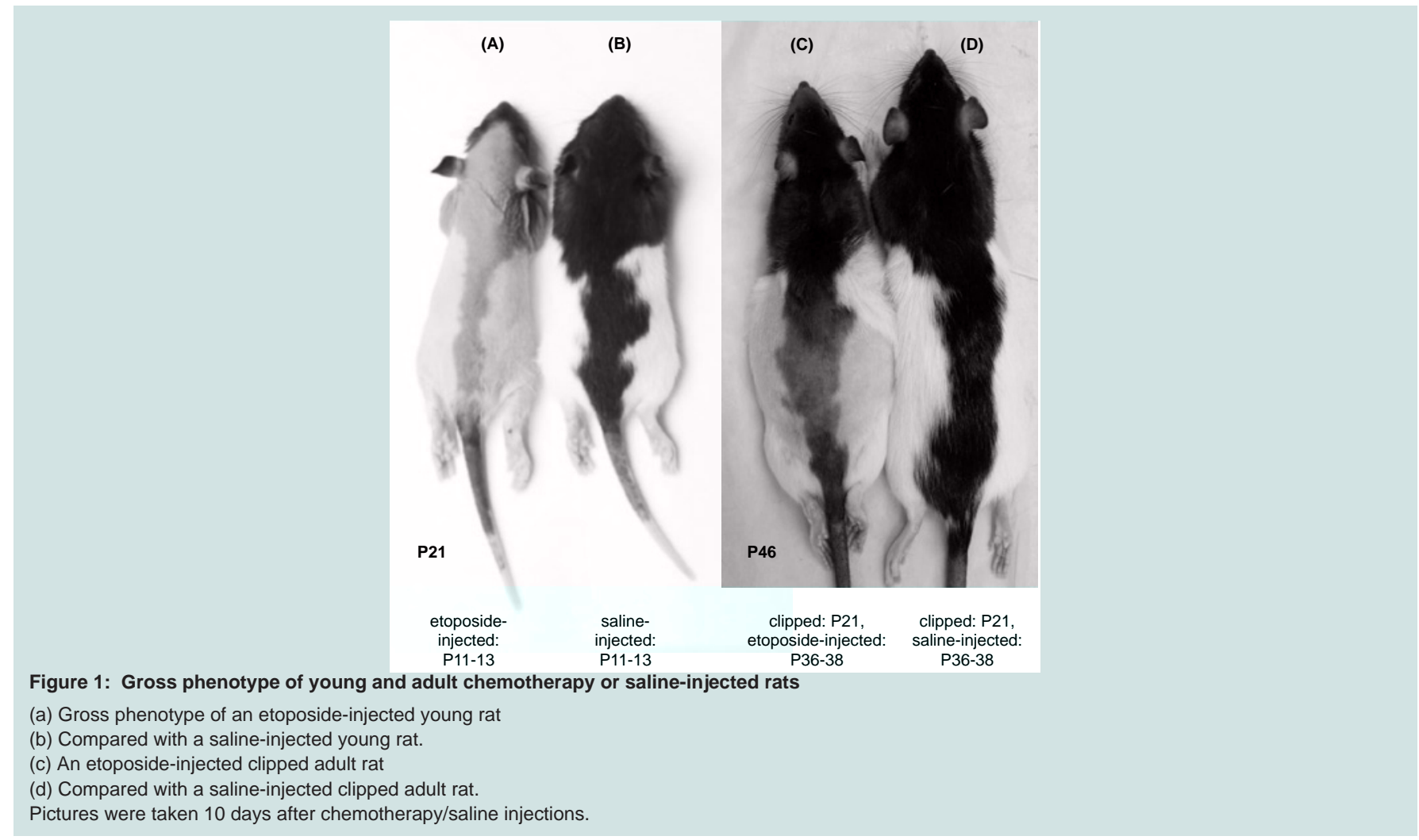


Citation: Villasante AC, Wikramanayake TC, Mauro LM, Yin NC, Elgart GW, et al. Comparison of Young and Adult Rat Models of ChemotherapyInduced Alopecia. J Clin Investigat Dermatol. 2014;2(2): 7.

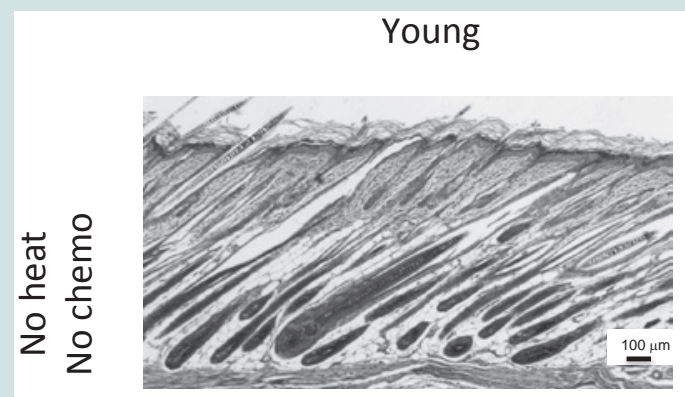

(A) P13 - No Injection Group 4

(B) P38 (clipped P21) - No Injection

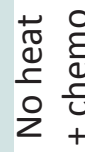

(C)

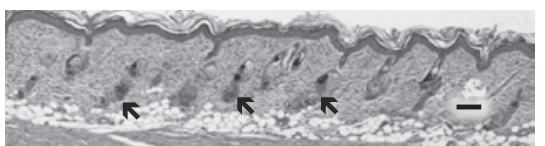

P21-Etop
Group 1

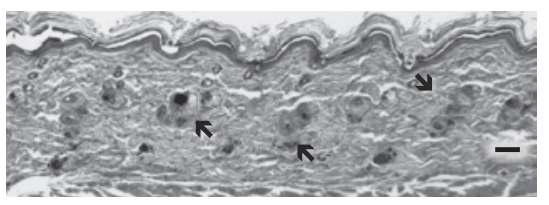

(D)
Group VI

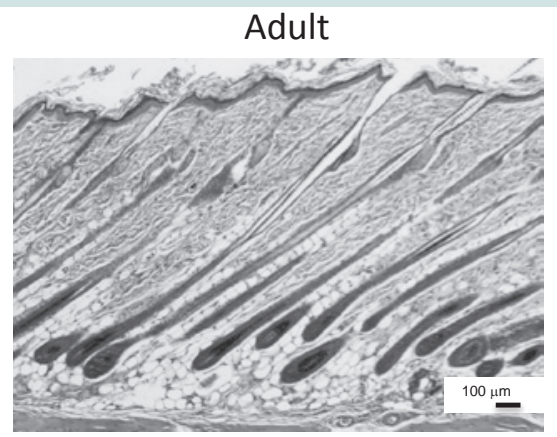

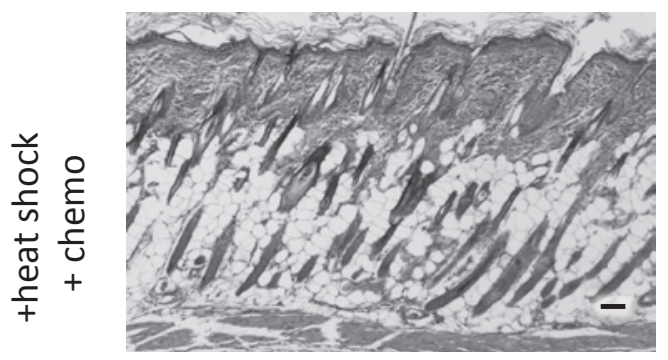

(E) P21 - Heat P9, Etoposide P11 13 Group 1

Figure 2: Histology of non-injected and chemotherapy-injected young and adult rat hair follicles

(a) Dorsal skin biopsies taken from non-injected young rats and

(b) non-injected clipped adult rats.

Pictures were taken at the time corresponding rats were receiving chemotherapy. Arrows point to whether hair shaft has penetrated or not, which is used to determine whether the HF is in anagen VI.

(c) Dorsal skin biopsies 10 days after etoposide-injection in the young rat and the

(d) Clipped adult.

Arrows point to HF dystrophy.

(e) Dorsal skin biopsies 10 days after etoposide-injection in young and

(f) Clipped adult rats that had received heat shock therapy prior to chemotherapy.

\section{Protection of anagen hair follicles against chemotherapy- induced alopecia}

Similar to our previous observations in the young and adult mouse models [11], topical heat preconditioning was found to confer HF protection against CIA in both the young and adult rat models (Table 1, Table 2). The effects of heat shock treatment were similar in both models. Macroscopically, both heat-treated models showed protection from CIA; in other words, both demonstrated preservation of body hair. Microscopically, both young and adult heat-treated models displayed abundant HSPA1A (HSP70) staining in the HFs and epidermis at the time of chemotherapy administration, as anticipated (Figure 3b, Figure 3d). Relative HSP levels were as follows: young heat-treated: 2.9+/-0.1 IOD; young control: 1.0+/-0.02 integrated optical density units (IOD) [18]; adult heat-treated: $3.1+/$ -
0.22 IOD; adult control: 0.9+/-0.1 IOD. Baseline HSPA1A expression was detected in the epidermis of the young control rat (Group 4) (Figure 3a), as observed before [11], but was not detected in the adult control rat skin (Figure 3c).

\section{Discussion}

We have established the gross and histological equivalence of the young and adult Long Evans rat models in response to etoposide and cyclophosphamide chemotherapy. We have also established gross and histological equivalence of the models in response to heat shock treatment, a therapy previously shown to protect from CIA $[11,12]$. Grossly, we observed that both models develop alopecia after administration of chemotherapy; however, they differ from each other in the pattern of alopecia, as we have previously observed [12]. 
Citation: Villasante AC, Wikramanayake TC, Mauro LM, Yin NC, Elgart GW, et al. Comparison of Young and Adult Rat Models of ChemotherapyInduced Alopecia. J Clin Investigat Dermatol. 2014;2(2): 7.

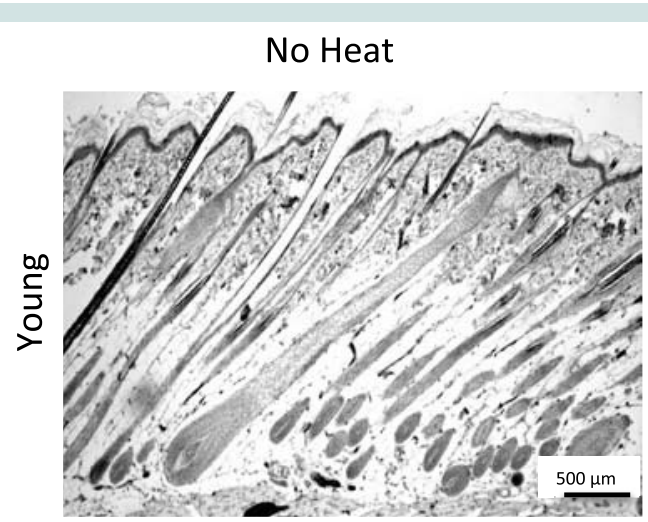

(A) P13- No Heat

Group 4

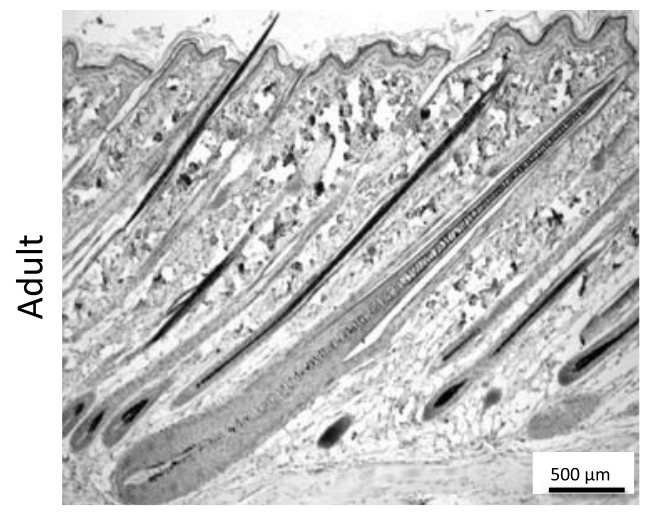

(C) P38 - No Heat

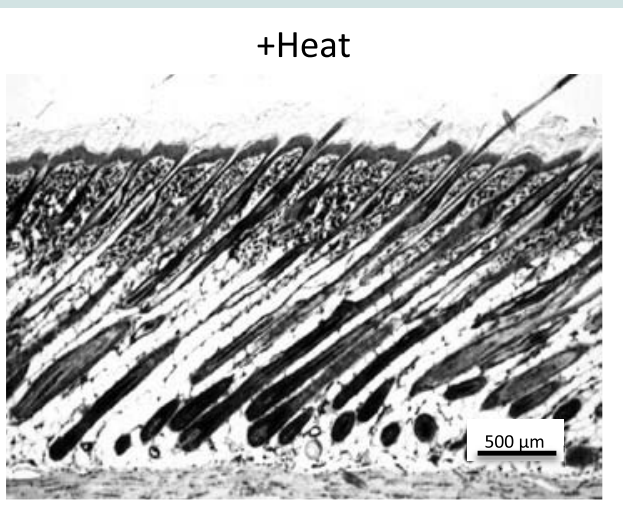

(B) P11 - Heat on P9 Group d

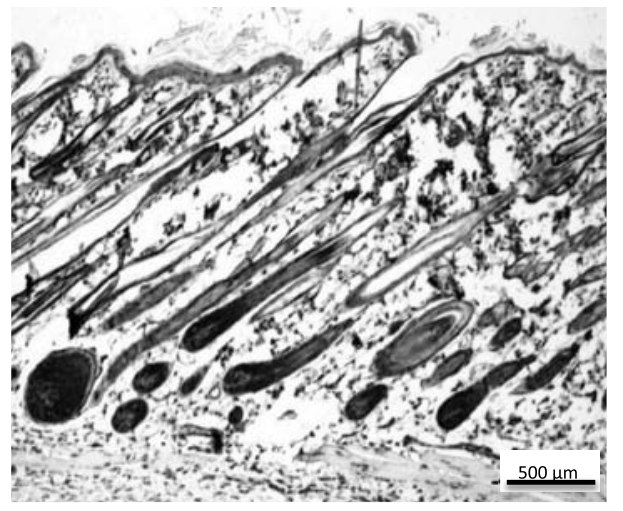

(D) P36- Heat on P34

Group D

Figure 3: Heat shock therapy in hair follicles of the young and adult rat models

Skin sections, immunostained for HspA1A (brown color), from the lower dorsal back of non-injected young rats that were (a) non-heat-treated versus (b) heattreated.

Skin sections, immunostained for HspA1A (brown color), from the lower dorsal back of non-injected adults that were (c) non-heat-treated and clipped versus (d) heat-treated and clipped.

The young model developed total body alopecia while the adult model developed circumscribed alopecia corresponding to the clipped area.

Histologically, at the time of chemotherapy administration, HFs in both young and adult rat models were similar in appearance. Both young and clipped adult rats contain follicles predominantly in anagen VI, based on criteria by Hendrix et al. [17]. This provides evidence that HFs in young and adult models are equally susceptible to alopecic chemotherapy. After administration of chemotherapy, HF in both the young and adult pigmented rats exhibited the dystrophic catagen response $[10,17,19]$. Our results show that cyclophosphamide, as well as etoposide, have a similar effect in pigmented rat HFs as they do in adult mouse HFs.

In addition, after administration of heat shock two days prior to chemotherapy, both young and adult rats demonstrate significant, comparable, and consistent protection from etoposide-induced and cyclophosphamide-induced alopecia. Heat shock treatment activates an intracellular stress protein (Hsp) response that protects cells within the hair follicle from the cytotoxic effects of chemotherapy [11] Heat shock was found to locally activate the stress protein response in noncancerous tissues without interfering with antineoplastic activity of chemotherapy in cancer cells [11].

Both models are useful in approximating the clinical and histological response of human scalp HFs to alopecic chemotherapy. In patients who develop CIA, proliferation of matrix cells in the bulb ceases abruptly and the hair shaft narrows and eventually breaks, leading to hair shedding, or effluvium, within 1-3 weeks after initial administration of chemotherapy [1]. Additionally, human HFs are pigmented, as are HFs in both the young and adult rat models used in this study; thereby, pigmented murine models offer an advantage over albino models in mimicking the human scalp.

The young model does not require manual induction of anagen, and it produces total body alopecia. A total body alopecic response is simpler to evaluate. Furthermore, because almost all (90\%) of human scalp HFs are in anagen at any given time, a total body alopecia better mimics the clinical appearance of CIA. Additionally, because the young model is synchronized in anagen for 14 days, it provides a wider window of treatment for protective compounds than the adult model. Lastly, the young rat is susceptible to transplantable Shay's 
Citation: Villasante AC, Wikramanayake TC, Mauro LM, Yin NC, Elgart GW, et al. Comparison of Young and Adult Rat Models of ChemotherapyInduced Alopecia. J Clin Investigat Dermatol. 2014;2(2): 7.

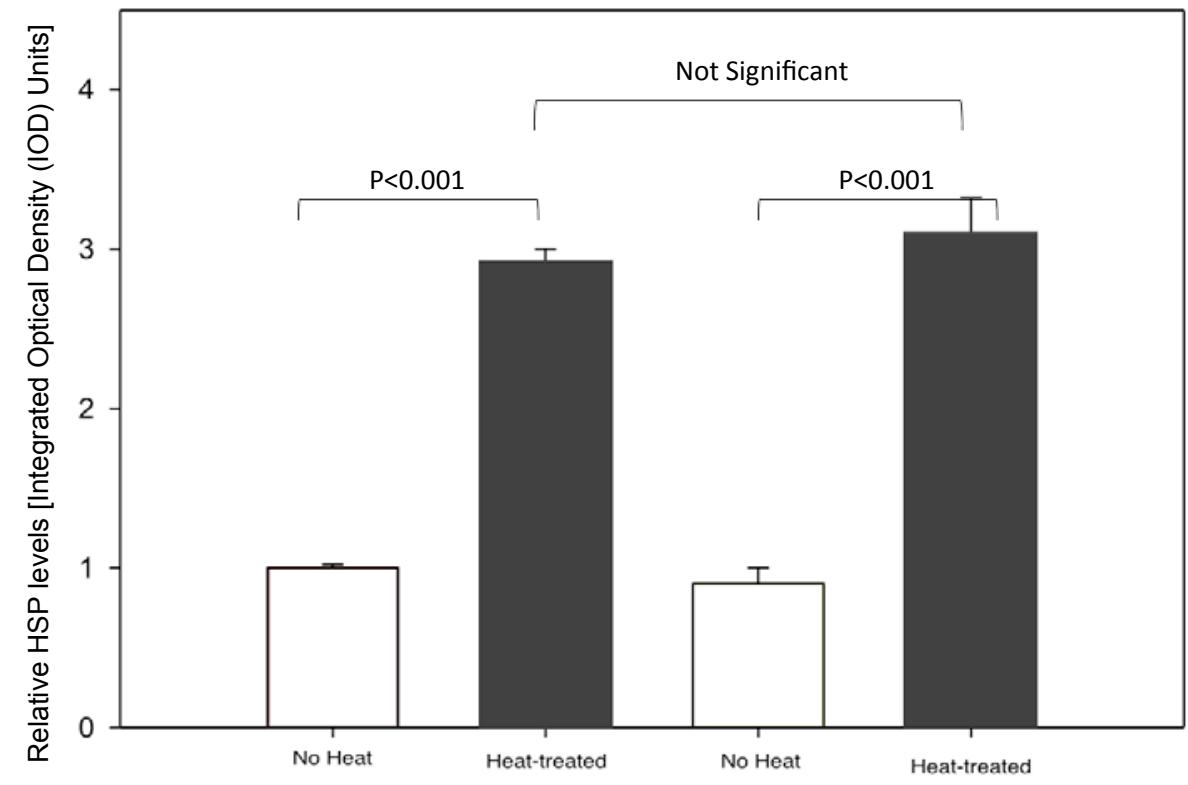

Young

Adult

Figure 4: Relative HSP levels after heat shock therapy in young and adult rat models

Relative HSP levels in IOD units for control and heat-treated groups in the young and adult rat models 48 hours after heat shock.

Chloroleukemia which induces chloromas and leukemias; this allows testing of whether the compound in question will have an effect on the efficacy of chemotherapy [9].

These aforementioned factors make the young pigmented rat more suitable model for initial screening of potential therapies. The adult pigmented rat is, then, a useful model to verify therapies after they have been screened in the young rat.

\section{References}

1. Trueb RM (2010) Chemotherapy-induced alopecia. Curr Opin Support Palliat Care 4: 281-284.

2. Wang J, Lu Z, Au JL (2006) Protection against chemotherapy-induced alopecia. Pharm Res 23: 2505-2514.

3. Miteva M, Misciali C, Fanti PA, Vincenzi C, Romanelli P, et al. (2011) Permanent alopecia after systemic chemotherapy: a clinicopathological study of 10 cases. Am J Dermatopathol 33: 345-350.

4. Tierney AJ, Taylor J, Closs SJ (1992) Knowledge, expectations and experiences of patients receiving chemotherapy for breast cancer. Scand J Caring Sci 6: 75-80.

5. Freedman TG (1994) Social and cultural dimensions of hair loss in women treated for breast cancer. Cancer Nurs 17: 334-341.

6. Munstedt K, Manthey N, Sachsse S, Vahrson H (1997) Changes in self- concept and body image during alopecia induced cancer chemotherapy. Support Care Cancer 5: 139-143.

7. Sun CC, Bodurka DC, Weaver CB, Rasu R, Wolf JK, et al. (2005) Rankings and symptom assessments of side effects from chemotherapy: insights from experienced patients with ovarian cancer. Support Care Cancer 13: 219-227.

8. Jayde V, Boughton M, Blomfield P (2013) The experience of chemotherapyinduced alopecia for Australian women with ovarian cancer. Eur J Cancer Care 22: 503-512.

9. Hussein AM, Jimenez JJ, McCall CA, Yunis AA (1990) Protection from chemotherapy-induced alopecia in a rat model. Science 249:1564-1566.

10. Paus R, Handjiski B, Eichmuller S, Czarnetzki BM (1994) Chemotherapyinduced alopecia in mice. Induction by cyclophosphamide, inhibition by cyclosporine A, and modulation by dexamethasone. Am J Pathol 144: 719734.

11. Jimenez JJ, Roberts SM, Mejia J, Mauro LM, Munson JW, et al. (2008) Prevention of chemotherapy-induced alopecia in rodent models. Cell Stress Chaperones 13: 31-38.

12. Wikramanayake TC, Amini S, Simon J, Mauro LM, Elgart G, et al. (2012) A novel rat model for chemotherapy-induced alopecia. Clin Exp Dermatol 37: 284-289.

13. Chase HB (1954) Growth of the hair. Physiol Rev 34: 113-126. 
Citation: Villasante AC, Wikramanayake TC, Mauro LM, Yin NC, Elgart GW, et al. Comparison of Young and Adult Rat Models of ChemotherapyInduced Alopecia. J Clin Investigat Dermatol. 2014;2(2): 7.

14. Van Scott EJ, Reinertson RP, Steinmuller R (1957) The growing hair roots of the human scalp and morphologic changes therein following amethopterin therapy. J Invest Dermatol 29: 197-204.

15. Whiting DA (1996) Chronic telogen effluvium: increased scalp hair shedding in middle-aged women. J Am Acad Dermatol 35: 899-906

16. Muller-Rover S, Handjiski B, van der Veen C, Eichmüller S, Foitzik K, et al (2001) A comprehensive guide for the accurate classification of murine hair follicles in distinct hair cycle stages. J Invest Dermatol 117: 3-15.

17. Hendrix S, Handjiski B, Peters EM, Paus R (2005) A guide to assessing damage response pathways of the hair follicle: lessons from cyclophosphamideinduced alopecia in mice. J Invest Dermatol 125: 42-51.

18. Xiao C, Chen S, Yuan M, Ding F, Yang D, et al. (2004) Expression of the 60 $\mathrm{kDa}$ and $71 \mathrm{kDa}$ heat shock proteins and presence of antibodies against the $71 \mathrm{kDa}$ heat shock protein in pediatric patients with immune thrombocytopenic purpura. BMC Blood Disord 4: 1.

19. Paus R, Haslam IS, Sharov AA, Botchkarev VA (2013) Pathobiology of chemotherapy-induced hair loss. Lancet Oncol 14: e50-59.

\section{Copyright}

(C) 2014 Villasante AC, et al. This is an open access article distributed under the Creative Commons Attribution License, which permits unrestricted use, distribution, and reproduction in any medium, provided the original work is properly cited.

\section{Acknowledgements}

We would like to thank the Locks of Love Foundation for their support. 